\title{
Dislocations as a boundary between charge density wave and oxygen rich phases in a cuprate high temperature superconductor
}

\author{
Nicola Poccia \\ Department of Physics, Harvard University, Cambridge, Massachusetts 02138, USA* \\ Alessandro Ricci \\ Deutsches Elektronen-Synchrotron DESY, Notkestrae 85, D-22607 Hamburg $\dagger^{\dagger}$ \\ Gaetano Campi \\ Institute of Crystallography, CNR, via Salaria Km 29.300, Monterotondo, Roma, 00015, Italy \\ Antonio Bianconi \\ RICMASS Rome International Center for Materials Science Superstripes, via dei Sabelli 119A, 00185 Roma, Italy; \\ Institute of Crystallography, CNR, via Salaria Km 29.300, Monterotondo, Roma, 00015, Italy; \\ National Research Nuclear University, MEPhI, Kashirskoe sh. 31, 115409, Moscow, Russi $\S^{\S}$
}

\begin{abstract}
Multiple functional ionic and electronic orders are observed in high temperature superconducting cuprates. The charge density wave order is one of them and it is spatially localized in spatial regions of the material. It is also known that the oxygen interstitials introduced by chemical intercalation self-organize in different oxygen rich regions corresponding with hole rich regions in the $\mathrm{CuO}_{2}$ layers left empty by the charge density wave order domains. However, what happens in between these two order is not known, and neither there is a method to control this spatial separation. Here we demonstrate by using scanning nano X-ray diffraction, that dislocations or grain boundaries in the material can act as boundary between charge density wave and oxygen rich phases in a optimally doped $\mathrm{La}_{2} \mathrm{CuO}_{4+y}$ high temperature superconductor. Dislocations can be used therefore to control the anti-correlation of the charge density wave order with the oxygen interstitials in specific portion of the material.
\end{abstract}

Dislocations or grain boundaries are known to change the properties of electronic materials [1] 2]. Although dislocations are often considered as a problem for the performances of a material, it is from the very beginning of semiconductor science that scientists tried to use them to engineer their functionalities [3. Currently, novel methods of fabrication to realize networks of dislocation have been adopted to change the electronic functionalities of Si 4. In the proximity of dislocations, the strain of a material is modulated [5. Strain (e.g. chemical pressure, microstrain, misfit-strain) is a material dependent parameter which have a deep influence in the electronic properties of the material and it has been proposed to play a crucial role also in the properties of high temperature superconductors [6] [7] as well for their phase diagram description 8 . In cuprates, since the spacer layer has a dissimilar lattice constants than the active layer, the mismatch typically results in the formation of strainrelieving misfit dislocations [9]. However, the search for an accurate description of the strain in strongly disordered systems is a current field of research [10] [11] [12] 13 .

Dislocations are a manifestation of a strongly disordered system and recently they have been exploited as

\footnotetext{
* npoccia@physics.harvard.edu

$\dagger$ phd.alessandro.ricci@gmail.com

$\ddagger$ gaetano.campi@ic.cnr.it

$\S$ antonio.bianconi@ricmass.eu
}

resistive switching in oxides [14, since they can act as bistable nanowires. The lattice can be sensitively different at the core of a dislocation [15], with oxygen that can get there pinned at elevated temperatures [16 and as a function of the annealing time. Materials under strain determine a collective motion of dislocations which give rise to strain burst difficult to control [17 [18. The role of dislocations as a fast route for oxygen diffusion is debated [19. Electro-switching transitions are observed in Mott insulators through the creation of metallic paths [20], which might involve oxygen-ion transport along nanoscale dislocation networks.

Evidence of nanoscale phase separation has recently been found in functional oxides like cobalates [21, bismutates [22] and in cuprates [23 26]. In cuprates, the oxygen interstitials (O-i) organization and the charge density wave $(\mathrm{CDW})$ ordering, coupled to the incommensurate periodic local lattice distortions (LLD), have been found to be spatially anti-correlated and to influence the macroscopic quantum condensate [27, 28, 29, 30, 31] 23, 24] 32 33 34 in a landscape of filamentary networks 35] [36] 25] 27] 37. In these works a non-euclidean geometry has been proposed to describe the optimal inhomogeneity of the cuprates [25] 27]. Non-euclidean geometries are also known to determine further complexity in the disposition and dynamics of defects [38. The nanoscale phase separation observed in the pnictides suggest the occurrence of dislocations at the interface [39]. Therefore, gaining control on the network described by the spatial arrangements of ordered defects is important 
(a)

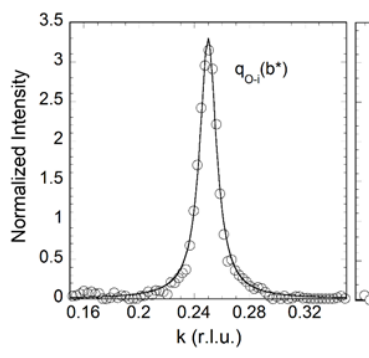

(d)

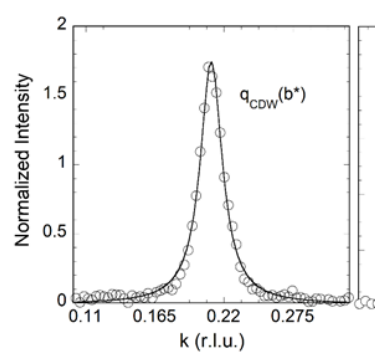

(b)

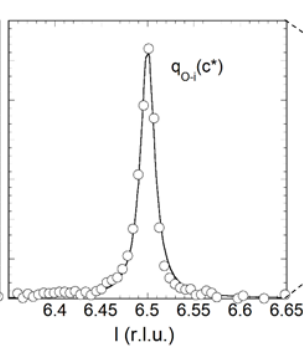

I (r.I.u.)

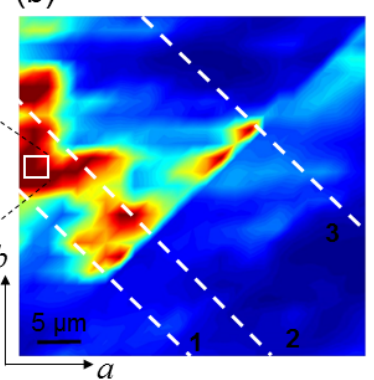

(e) (c)

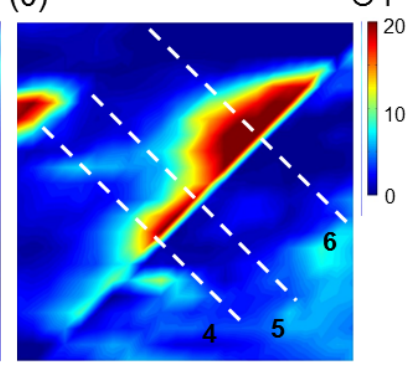

(f)

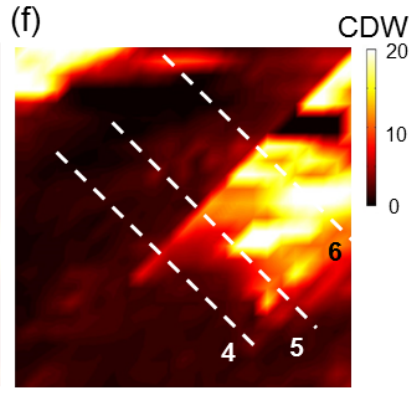

FIG. 1. Panel (a) shows the representative X-ray scattering profiles of a typical O-i satellite due to ordered oxygen-interstitials with wave vector $\mathrm{q}_{O-i}$. The solid lines are the Lorentzian best fit curves. The panels (b) and (c) show the xy map of the intensity of the $\mathrm{O}-\mathrm{i}$ satellite in different portions of the sample. Two different diagonal dislocations which induce sharp boundaries are shown. Here the color goes from dark blue to dark red as indicated in the O-i color bar. Panel (d) shows representative scattering profiles of the satellite due to charge density wave order with wave-vector qCDW. Panel (e) and (f) show the xy mapping of the intensity of the CDW satellite in the same spatial portions shown in panels respectively (b) and (c). Here the color goes from dark red to white as indicated in the CDW color bar. Visual inspection of the images shows that close to the diagonal linear boundary, the CDW or O-i order abruptly changes. Comparing panels (b) and (c) with the panels (e) and (f), it is clear that the dislocation plays the role of boundary between the O-i rich and CDW rich regions of the sample. The bar is 5 micron.

for the design of materials [40]. Indeed, the collective motion of the cooper pairs can be strongly influenced by an annealed complex network [41. The control of defects is considered a central problem in advanced materials and atomic heterostructure [42], and in the realization of array of artificial atoms in strongly correlated systems [43]. Recently, the precise control of disorder in an array of vortices, which behave as an array of superatoms, has made possible the observation of a dynamic vortex Mott insulator to metal transition [44].

Here we show that dislocations can act as boundaries between charge density waves and oxygen rich puddles in a cuprate superconductor. We use scanning nano Xray diffraction to image the spatial organization of the oxygen interstitials and charge density wave across the dislocation boundary of a cuprate superconductor.

\section{EXPERIMENTAL METHOD}

The sample of $\mathrm{La}_{2} \mathrm{CuO}_{4}$ was grown first by flux method and then doped by electrochemical oxidation. The critical superconducting temperature of the oxygen doped
$\mathrm{La}_{2} \mathrm{CuO}_{4+y}(y=0.12)$ was determined to be $41 \mathrm{~K}$ by single coil resistivity measurements. The orthorhombic lattice parameters of single crystal were determined to be $\mathrm{a}=(5.386 \pm 0.004) \AA$, $\mathrm{b}=(5.345 \pm 0.008) \AA$, $\mathrm{c}=(13.205$ $\pm 0.031) \AA$ at room temperature. The space group of the sample is Fmmm. Nano X-ray diffraction experiments were performed on the ID13 beam line of ESRF, using a photon energy of $14 \mathrm{keV}$ focused on a $300 \times 300 \mathrm{~nm}^{2}$ spot on the sample a-b surface. X-Ray Diffraction (XRD) patterns were collected in reflection geometry to record the reflections on the $\mathrm{b}^{*} \mathrm{c}^{*}$ reciprocal plane by a Frelon area detector. The scanning step was set to $3 \mu \mathrm{m}$ in both horizontal and vertical directions. The experiment has been performed at room temperature. Thanks to the high brilliance source, it has been possible to record a large number of weak superstructural peaks around the main Bragg peaks of the average structure.

\section{RESULTS AND DISCUSSION}

Optimally doped $\mathrm{La}_{2} \mathrm{CuO}_{4+y}$ is ideal for the investigation of interstitials diffusion in copper oxides because 
the $\mathrm{O}-\mathrm{i}$ are mobile in the $\mathrm{La}_{2} \mathrm{O}_{2+y}$ layers intercalated between the superconducting $\mathrm{CuO}_{2}$ planes.

The main Bragg peaks of the single crystal are surrounded by the known satellite peaks with wave-vector $\mathrm{q}_{O-i}=0.09 \mathrm{a}^{*}+0.25 \mathrm{~b}^{*}+0.5 \mathrm{c}^{*}$, due to the O-i dopants ordered in three-dimensional superstructure (see Fig. 1). Alongside the $\mathrm{O}-\mathrm{i}$ peaks, indexing of superlattice peaks around the Bragg lattice reflections show the presence of incommensurate modulations $\mathrm{q}_{C D W}=0.035 \mathrm{a}^{*}+0.21 \mathrm{~b}^{*}$ $+0.29 \mathrm{c}^{*}$ (see Fig. 1). These modulations are charge density waves strongly coupled with the incommensurate periodic local lattice distortions (LLD) of the copper plane 29] 24] 25, forming stripes running in the a direction with a correlation length of about $2.6 \mathrm{~nm}$ in the $\mathrm{b}$ direction. The correlation between CDW and LLD is know from long time in layered materials 45 . These incommensurately modulated LLDs could define the nanoscale organization of CDW domains that show average size of $12 \mathrm{~nm}$. The $\mathrm{X}$ ray diffraction profiles of the two satellite reflections, $\mathrm{q}_{O}-{ }_{i}$ and $\mathrm{q}_{C D W}$ in the $\mathrm{b}^{*} \mathrm{c}^{*}$ plane are shown in Fig. 1. The average increase in the intensity of the qCDW is observed below $250 \mathrm{~K}$ until reaches a maximum at about $100 \mathrm{~K}[36$.

The spatial distribution of both the O-i and the CDW satellites has been studied by using the X-ray micro diffraction apparatus at the ESRF. Scanning the sample using nanometer piezo-stages we recorded the spatial dependence of the O-i and CDW peak intensity. The resulting spatial maps show clear dislocations on the a-b plane of the crystal. Figure 1 shows two typical dislocation running on the $\mathrm{a}-\mathrm{b}$ plane. Its deepness along the c-axis overcome the X-ray penetration length, while its largeness on the a-b plane extends for less than the beam size $(300 \times 300) \mathrm{nm}$.

Our key experimental discovery is that such dislocation can control both the arrangement of the O-i and CDW domains in real space. It shows that region of CDW higher order and larger clusters are formed on the bottom portion of the image close to the dislocation boundary. On the top part, instead, the O-i order is dominant. More specifically, we observe the enhancement of O-i and the CDW melting in proximity of the dislocation. The intensity profiles along the white six dashed lines of the maps in Fig. 1 are shown in Fig. 2, and highlight the anti-correlated spatial arrangement of the two phases O-i and CDW in the proximity of the dislocation.

The general character of this spatial anti-correlation results evident also from the scatter plot of $\mathrm{O}-\mathrm{i}$ intensity versus CDW intensity (Fig. 3) calculated on the whole maps of Fig. 1. As O-i intensity becomes higher, the CDW intensity decreases, and vice versa. The observed dislocations determine a physical barrier for the oxygen interstitial diffusivity [46. The dislocation can be used to manipulate the ions distribution which is considered a relevant problem in modern material science [47. The concentration of the O-i order on one side the dislocation creates a CDW order on the other side of the dislocation. This artificial separation induced by the dislocation de-
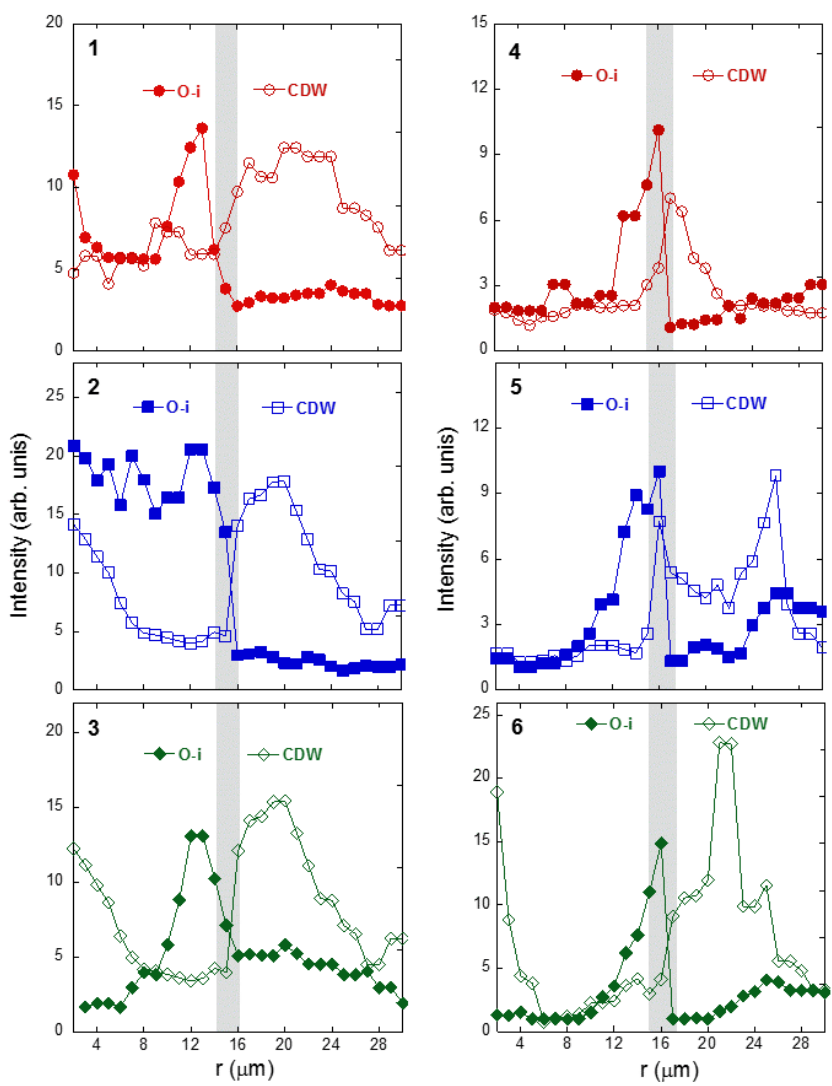

FIG. 2. The profile cut of the integrated intensity map of the $\mathrm{q}_{O-i}$ and $\mathrm{q}_{C D W}$ peak are shown along the dashed white lines as indicated in the maps of Fig. 1. Each panel shows respectively the $\mathrm{O}-\mathrm{i}$ and $\mathrm{CDW}$ intensity along the profile cuts indicated with the numbers from 1 to 6 . The grey area is the region occupied by the dislocation. It is visible the anticorrelation of the $\mathrm{O}-\mathrm{i}$ and $\mathrm{CDW}$ orders in the proximity of the dislocation.

termines a more pronounced spatial anti-correlation between the O-i and the CDW orders.

Recently attention on the control and properties of dislocations has been given in van der Waals heterostructures 48 through the observation of atomic scale ripples. Although in the case we report here the dislocation is observed in a bulk crystal, the observed pinning of anticorrelated phases could be basically explained on how the material accommodate the high load of natural strain. Modulation of the local strain in the proximity of the dislocation can determine the observed oxygen ordering [49. Elastic field engineering is considered important in many field of science, especially focussing on the realization of high - strength materials [50. The possibility of modulate the strain field creating dislocations could determine the occurrence of the CDW and O-i rich domains. It has been suggested that bulk dislocations can modulate the layered solid into ripples and that this could be 


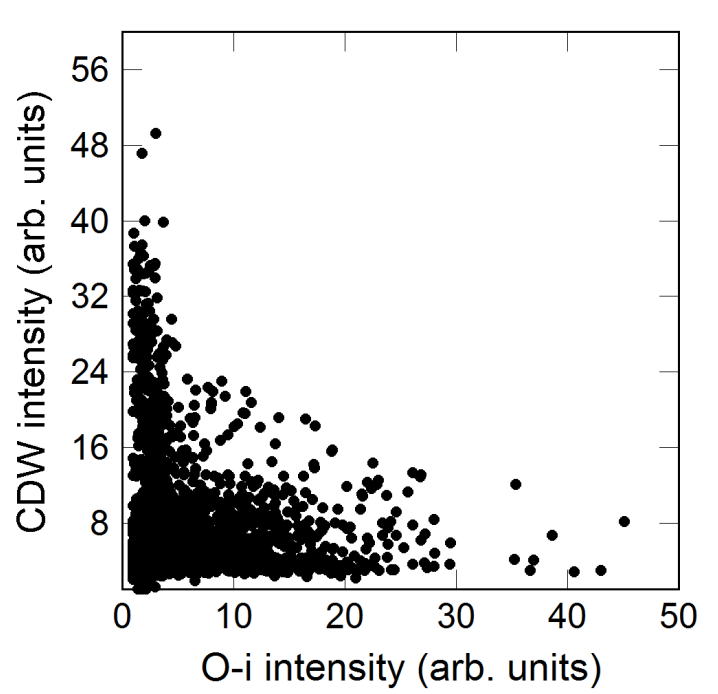

FIG. 3. The anti-correlation is further quantified by plotting the intensity of the whole maps for $\mathrm{q}_{C D W}$ as a function of $\mathrm{q} O-i$. The observed scatter plots demonstrate the spatial anti-correlation between the two phases near the boundary.

more common in materials than expected [51]. The modulation of the copper plane as ripples (e.g. short range periodic local lattice distortions) close to the dislocation observed in our experiment, can explain the observed pinning of the charge density wave order. However, it is also possible to discuss the results reported in this paper in terms of a grain boundary separating the CDW and the $\mathrm{O}-\mathrm{i}$ rich domains. It is known indeed that grain boundaries can give to the material unique electronic properties different from that of the bulk [2].

In conclusion, we have performed a scanning nano $\mathrm{X}$-ray diffraction experiment and we have imaged the spatial dependence of the ordered $\mathrm{O}$-i domains and the CDW rich domains in the proximity of a several tens micrometer and diagonal sized dislocations. We observe that the each dislocation acts as a boundary between the short range incommensurate charge density wave in the $\mathrm{CuO}_{2}$ plane, and the oxygen interstitials rich domains in the spacer layer corresponding with neighbor filamentary high density metallic wires in the $\mathrm{CuO}$ plane. The observed spatial anti-correlation can be exploited in bistable switching devices thanks to the separation between the two phases. The control of dislocations is therefore an approach to control the boundaries of phase separation between $\mathrm{O}$-i rich metallic filamentary domains and CDW rich domain in cuprate oxide superconductors in the so called superstripes landscape 52.

\section{ACKNOWLEDGEMENTS}

The work was supported by the Italian Ministry of Education and Research, the Dutch FOM and NWO foundations and Superstripes Institute. We thank ESRF synchrotron facility for support. In particular we thank Manfred Burghammer and the staff of the ID13 beamline for experimental help.
[1] Setter, N. and Waser, R., Electroceramic materials. Acta Mater. 48, 151 (2000).

[2] Hilgenkamp, H. and Mannhart, J. Grain boundaries in high- $T_{c}$ superconductors. Rev. Mod. Phys., 74, 485-549 (2002).

[3] Calzecchi, F., Gondi, P., and Schintu, F. Bilateral microscopy of dislocations and dislocation diodes in germanium. Il Nuovo Cimento B 58, 376-380, (1968).

[4] Kittler, M., Yu, X., Mchedlidze, T., Arguirov, T., Vyvenko, O. F., Seifert, W., Reiche, M., Wilhelm, T., Seibt, M., Vo, O., Wolff, A., and Fritzsche, W. Regular dislocation networks in silicon as a tool for nanostructure devices used in optics, biology, and electronics. Small, 3, 964-973, (2007).

[5] Davletova, A., and Ulyashin, A. Strain-induced modulation of band structure of silicon. Journal of Applied Physics, 104 (2), 024501, (2008).

[6] Poccia, N., Ricci, A., and Bianconi, A. (2010). Misfit strain in superlattices controlling the Electron-Lattice interaction via microstrain in active layers. Advances in Condensed Matter Physics, 2010 , 1-7, (2010).

[7] Bianconi, A., Saini, N. L., Agrestini, S., Di Castro, D., and Bianconi, G. The strain quantum critical point for superstripes in the phase diagram of all cuprate perovskites. International Journal of Modern Physics B, 14,
3342-3355 (2000).

[8] Kugel, K. I., Rakhmanov, A. L., Sboychakov, A. O., Kusmartsev, F. V., Poccia, N., and Bianconi, A. A two-band model for the phase separation induced by the chemical mismatch pressure in different cuprate superconductors. Superconductor Science and Technology, 22, 014007, (2009).

[9] Venables, J. A., Spiller, G. D. T., and Hanbucken, M. Nucleation and growth of thin films. Reports on Progress in Physics, 47, 399-459 (1984).

[10] Egami, T. Atomic level stresses. Progress in Materials Science, 56, 637-653, (2011).

[11] Campi, G., Cappelluti, E., Proffen, T., Qiu, X., Bozin, E. S., Billinge, J. L. S., Agrestini, S., Saini, N. L., Bianconi, A., Study of temperature dependent atomic correlations in $\mathrm{MgB}_{2}$. The European Physical Journal B, 52, 15 (2006).

[12] Bozin, E. S., Huq, A., Shen, B., Claus, H., Kwok, W. K.,and Tranquada, J. M. Charge-screening role of c-axis atomic displacements in YBCO and related superconductors. Phys. Rev. B, 93, 054523 (2016).

[13] Campi, G., Ricci, A., Bianconi, A., Local Structure in $M g_{1-x} A l_{x} B_{2}$ System by High Resolution Neutron Diffraction. Journal of Superconductivity and Novel Magnetism, 25 , 1319-1322 (2012). 
[14] Szot, K., Speier, W., Bihlmayer, G., and Waser, R. Switching the electrical resistance of individual dislocations in single-crystalline $\mathrm{SrTiO}_{3}$. Nature Materials, 5, 312-320, (2006).

[15] Jia, C. L., Thust, A., and Urban, K. Atomic-scale analysis of the oxygen configuration at a 3 dislocation core. Phys. Rev. Lett., 95, 225506+ . (2005).

[16] Senkader, S., Wilshaw, P. R., and Falster, R. J. Oxygendislocation interactions in silicon at temperatures below $700 C$ : Dislocation locking and oxygen diffusion. Journal of Applied Physics, 89, 4803-4808, (2001).

[17] Miguel, M.-C., Vespignani, A., Zapperi, S., Weiss, J., and Grasso, J.-R. Intermittent dislocation flow in viscoplastic deformation. Nature, 410 (6829), 667-671, (2001).

[18] Csikor, F. F., Motz, C., Weygand, D., Zaiser, M., and Zapperi, S. Dislocation avalanches, strain bursts, and the problem of plastic forming at the micrometer scale. Science, 318 (5848), 251-254 (2007).

[19] Metlenko, V., Ramadan, A. H. H., Gunkel, F., Du, H., Schraknepper, H., Hoffmann-Eifert, S., Dittmann, R., Waser, R., and De Souza, R. A. Do dislocations act as atomic autobahns for oxygen in the perovskite oxide $\mathrm{SrTiO}_{3}$ ? Nanoscale, 6 (21), 12864-12876 (2014).

[20] Guiot, V., Cario, L., Janod, E., Corraze, B., Ta Phuoc, V., Rozenberg, M., Stoliar, P., Cren, T., and Roditchev, D. Avalanche breakdown in $\mathrm{GaTa}_{4} \mathrm{Se}_{8-x} \mathrm{Te}_{x}$ narrowgap Mott insulators. Nature Communications, 4, 1722 (2013).

[21] Drees, Y.. et al. Hour-glass magnetic excitations induced by nanoscopic phase separation in cobalt oxides. Nature Communications 5, 5731+ (2014).

[22] Giraldo-Gallo, P. et al. Stripe-like nanoscale structural phase separation in superconducting BaPb1?xBixO3. Nature Communications 6, 8231+ (2015).

[23] Fratini, M. et al. Scale-free structural organization of oxygen interstitials in $\mathrm{La}_{2} \mathrm{CuO}_{4+y}$. Nature 466, 841-844 (2010).

[24] Poccia, N., et al. Optimum inhomogeneity of local lattice distortions in $\mathrm{La}_{2} \mathrm{CuO} \mathrm{O}_{4+y}$. Proc. Nat. Acad. Sci. USA 109, 15685-15690 (2012).

[25] Campi, G., et al., Inhomogeneity of charge-density-wave order and quenched disorder in a high-Tc superconductor. Nature 525, 359-362 (2015).

[26] Carlson, E. W. Condensed-matter physics: charge topology in superconductors. Nature 525, 329-330 (2015).

[27] Campi, G., Bianconi, A., High-temperature superconductivity in a hyperbolic geometry of complex matter from nanoscale to mesoscopic scale. Journal of Superconductivity and Novel Magnetism 29, 627-631 (2016).

[28] Kusmartsev, F.V., Di Castro, D., Bianconi, G., Bianconi, A., Transformation of strings into an inhomogeneous phase of stripes and itinerant carriers Physics Letters A 275, 118-123 (2000)

[29] Bianconi, A. et al. Coexistence of stripes and superconductivity: Tc amplification in a superlattice of superconducting stripes Physica C: Superconductivity 341, 1719$1722(2000)$

[30] Zaanen, J. High-temperature superconductivity: The benefit of fractal dirt. Nature 466, 825-827 (2010).

[31] Littlewood, P.B. Superconductivity: An x-ray oxygen regulator. Nature Materials 10, 726-727 (2011).

[32] Poccia, N., Ricci, A. and Bianconi, A. Fractal structure favoring superconductivity at high temperatures in a stack of membranes near a strain quantum critical point.
Journal of Superconductivity and Novel Magnetism 24, 1195-1200 (2011).

[33] Poccia, N. et al. Evolution and control of oxygen order in a cuprate superconductor. Nature Materials 10, 733-736 (2011).

[34] Saarela, M., Kusmartsev, F.V., Phase transitions to dipolar clusters and charge density waves in high tc superconductors. Preprint arXiv:1608.08081 (2016). URL http://arxiv.org/abs/1608.08081

[35] Ricci, A., Poccia, N., Campi, G., Coneri, F., Barba, L., Arrighetti, G., Polentarutti, M., Burghammer, M., Sprung, M., Zimmermann, M., and Bianconi, A. Networks of superconducting nano-puddles in $1 / 8$ doped $\mathrm{YBa}_{2} \mathrm{Cu}_{3} \mathrm{O}_{6.5+y}$ controlled by thermal manipulation. New Journal of Physics, 16 (5), 053030 (2014).

[36] Poccia, et al. Competing striped structures in $L a_{2}$ $\mathrm{CuO}_{4}+y$ J. Supercond. Nov. Magn. 26, 2703-2708 (2013).

[37] Yu, H., He, G., Lin, Z., Kusmartseva, A., Yua, J., Zhu, B., Yang, Y.-f., Xiang, T., Li, L., Wang, J., Kusmartsev, F.V., Jin, K., A conducting nano-filament (CNF) network as a precursor to the origin of superconductivity in electron-doped copper oxides. Preprint arXiv:1610.04788 (2016) URL http://arxiv.org/abs/1610.04788

[38] Lipowsky, P., Bowick, M. J., Meinke, J. H., Nelson, D. R., and Bausch, A. R. Direct visualization of dislocation dynamics in grain-boundary scars. Nature Materials, 4 , 407-411 (2005).

[39] Ricci, A., Poccia, N., Joseph, B., Innocenti, D., Campi, G., Zozulya, A., Westermeier, F., Schavkan, A., Coneri, F., Bianconi, A., Takeya, H., Mizuguchi, Y., Takano, Y., Mizokawa, T., Sprung, M., and Saini, N. L. Direct observation of nanoscale interface phase in the superconducting chalcogenide with intrinsic phase separation. Phys. Rev. B, 91, 020503 (2015).

[40] Aidhy, D. S., and Weber, W. J. Microstructure design for fast oxygen conduction. Journal of Materials Research, 31 (01), 2-16, (2015).

[41] Bianconi, G., Superconductor-insulator transition on annealed complex networks. Phys. Rev. E, 85, 061113+ (2012).

[42] Boschker, H., and Mannhart, J. Quantum-Matter heterostructures, Preprint arxiv: 1607.07239 (2016).

[43] Mannhart, J., Boschker, H., Kopp, T., and Valenti, R. Artificial atoms based on correlated materials. Reports on Progress in Physics, 79, 084508 (2016).

[44] Poccia, N. et al. Critical behavior at a dynamic vortex insulator-to-metal transition. Science 349, 1202-1205 (2015).

[45] Wilson, J. A., Di Salvo, F. J., and Mahajan, S. Chargedensity waves and superlattices in the metallic layered transition metal dichalcogenides. Advances in Physics, 24, 117-201 (1975).

[46] Chroneos, A., Yildiz, B., Tarancn, A., Parfitt, D., and Kilner, J. A. Oxygen diffusion in solid oxide fuel cell cathode and electrolyte materials: mechanistic insights from atomistic simulations. Energy and Environmental Science, 4, 2774 (2011).

[47] Kalinin, S. V., and Spaldin, N. A. Functional ion defects in transition metal oxides. Science, 341, 858-859 (2013).

[48] Kushima, A., Qian, X., Zhao, P., Zhang, S., and Li, J. Ripplocations in van der waals layers. Nano Lett., 15, 1302-1308 (2015). 
[49] Meng, Q. P., Welch, D. O., and Zhu, Y. Strain-induced changes of oxygen ordering in YBCO cuprates. Phys. Rev. B, 79, 134531+ (2009).

[50] Zhu, T., and Li, J. Ultra-strength materials. Progress in Materials Science, 55, 710-757 (2010).
[51] Gruber, J., Lang, A. C., Griggs, J., Taheri, M. L., Tucker, G. J., and Barsoum, M. W. Evidence for bulk ripplocations in layered solids. Scientific Report, 6, 33451+ (2016).

[52] Bianconi, A. Superstripes Int. Journal of Modern Physics B, 14, 3289-3297 (2000). 